\title{
A Method of Calculation of Integrals of Products of Radial Atomic Wave Functions
}

\section{By Stephan Ormonde and Jerald S. Dick}

Abstract. A simple computational procedure for the calculation of certain useful integrals in atomic physics is described. The procedure has been compared with other techniques and was found to be superior.

1. Introduction. The numerical integration of radial integrodifferential equations encountered in some problems of atomic physics invariably requires the computation of functions consisting of integrals over products of radial atomic wave functions $(1,2)$. In this paper, we describe a simple iterative procedure for the construction and evaluation of such functions by a computer. The procedure is applicable whenever the radial wave functions are known in analytic form. Our method is more accurate and computationally faster than the quadrature procedures usually employed. It also permits the computation of the functions at any point of the integration grid without requiring the cumulative values at the preceding grid points.

The present procedure was originally developed for use in a computer program applied to a problem in scattering theory [3]. The radial equations which must be solved to determine the cross sections for the scattering of low energy electrons by atoms are defined by Percival and Seaton [4]. In a slightly different notation, these equations can be written

$$
\frac{d^{2} F_{i}(r)}{d r^{2}}=\sum_{j}\left[V_{i j}(r)-W_{i j}(r)\right] F_{j}(r) .
$$

The first term on the right-hand side of equation (1) is the so-called "direct potential" defined by

$$
V_{i j}^{L}(r)=-\frac{\delta_{i j}}{r}+\sum_{\lambda} f_{\lambda}\left(l_{1} l_{2} l_{1}^{\prime} l_{2}^{\prime} ; L\right) y_{\lambda}\left(P_{n l_{1}} P_{n^{\prime} l_{1^{\prime}}} ; r\right)
$$

with

$$
\begin{aligned}
y_{\lambda}\left(P_{n l_{1}} P_{n^{\prime} l_{1^{\prime}}} ; r\right)=\frac{1}{r^{\lambda+1}} \int_{0}^{r} x^{\lambda} P_{n l_{1}}(x) P_{n^{\prime} l_{1^{\prime}}}(x) d x & \\
& +r^{\lambda} \int_{r}^{\infty} x^{-\lambda-1} P_{n l_{1}}(x) P_{n^{\prime} l_{1}}(x) d x,
\end{aligned}
$$

where

$$
f_{\lambda}\left(l_{1} l_{2} l_{1}^{\prime} l_{2}^{\prime} ; L\right)=\left(l_{1} l_{2} L\left|P_{\lambda}\left(\hat{r}_{1} \cdot \hat{r}_{2}\right)\right| l_{1}^{\prime} l_{2}^{\prime} L\right) .
$$

(See [4] for definition of the symbols.) We are interested in the computation of the "potential integrals" defined by equation (3).

Received November 30, 1964. Work supported jointly by the Lockheed Independent Research Program and the Air Force Special Weapons Center under Contract AF 29(601)-6171. 
2. The Iterative Procedure. The necessary recurrence formulae are derived below for the simple case of the hydrogen atom. For simplicity, consider only the first integral on the right-hand side of equation (3)

$$
y_{1}=\int_{0}^{r} x^{\lambda} P_{n l}(x) P_{n^{\prime} l^{\prime}}(x) d x .
$$

The functions $P_{n l}(x)$ are defined by

$$
\begin{aligned}
P_{n l}(x) & =x R_{n l}(x), \\
R_{n l}(x) & =-\left\{\left(\frac{2 z}{n}\right)^{3} \frac{(n-l-1) !}{2 n[(n+l) !]^{3}}\right\}^{1 / 2} e^{-(z / n) x}\left(\frac{2 z}{n} x\right)^{l} L_{n+l}^{2 l+1}\left(\frac{2 z}{n} x\right), \\
L_{n+l}^{2 l+1}\left(x^{\prime}\right) & =\sum_{k=0}^{n-l-1} \frac{(-1)^{k+1}[(n+l) !]^{2}\left(x^{\prime}\right)^{k}}{k !(n-l-1-k) !(2 l+1+k) !}, \quad x^{\prime}=\frac{2 z}{n} x
\end{aligned}
$$

(see [5]). Substituting these expressions in equation (4) and collecting the constants, we have

$$
y_{I}=k_{v v^{\prime}} \int_{0}^{r} d x x^{\lambda+2+l+l^{\prime}} e^{-\alpha x}\left(\sum_{k=0}^{n-l-1} a_{k} x^{k}\right)\left(\sum_{k=0}^{n^{\prime}-l^{\prime}-1} b_{k^{\prime}} x^{k^{\prime}}\right),
$$

where $\nu \equiv n l, \nu^{\prime} \equiv n^{\prime} l^{\prime}, \alpha=z\left(1 / n+1 / n^{\prime}\right)$,

$$
\begin{aligned}
k_{v v^{\prime}} & =\left(\frac{2 z}{n}\right)^{l+3 / 2}\left(\frac{2 z}{n^{\prime}}\right)^{l^{\prime}+3 / 2}\left\{\frac{(n-l-1) !\left(n^{\prime}-l^{\prime}-1\right) !(n+l) !\left(n^{\prime}+l^{\prime}\right) !}{4 n n^{\prime}}\right\}^{1 / 2} \\
a_{k} & =\frac{(-1)^{k+1}\left(\frac{2 z}{n}\right)^{k}}{k !(2 l+1+k) !(n-l-1-k) !}
\end{aligned}
$$

and similarly for $b_{k^{\prime}}$.

Now let $m=n-l-1, m^{\prime}=n^{\prime}-l^{\prime}-1$, and $q=\lambda+2+l+l^{\prime}$. For a given pair of atomic states, $m$ and $m^{\prime}$ are defined uniquetly, and the possible values of $\lambda$ are determined by the usual selection rules on the matrix elements [4]. Write the integral in equation (5) in the form

$$
I=\int_{0}^{r} e^{-\alpha x} x^{q} d x\left(c_{0}+c_{1} x+\cdots+c_{p} x^{p}\right)
$$

where

$$
\begin{aligned}
& c_{0}=a_{0} b_{0}, \quad c_{p}=a_{m} b_{m^{\prime}}, \quad p=m+m^{\prime}, \\
& c_{1}=a_{0} b_{1}+a_{1} b_{0}, \text { etc. }
\end{aligned}
$$

The integrals in equation (6) are all of the form

$$
I_{\mu}=\int_{0}^{r} e^{-\alpha x} x^{\mu} d x
$$

with the recurrence relation

$$
I_{\mu}=-\frac{1}{\alpha} e^{-\alpha r} r^{\mu}+\frac{\mu}{\alpha} I_{\mu-1}, \quad \mu \geqq 1,
$$




$$
I_{0}=\frac{1}{\alpha}\left(1-e^{-\alpha r}\right)
$$

The evaluation of $y_{\lambda}$ can now proceed in a number of ways.

Applying equation (7) to equation (6), we have

$$
\begin{aligned}
& I= c_{p} I_{p+q}+c_{p-1} I_{p+q-1}+\cdots+c_{0} I_{q} \\
&=-\frac{1}{\alpha} e^{-\alpha r}\left\{c_{p} r^{p+q}+\left(c_{p} \frac{p+q}{\alpha}+c_{p-1}\right) r^{p+q-1}+\cdots\right. \\
&+\left(\left(c_{p} \frac{p+q}{\alpha}+c_{p-1}\right) \frac{p+q-1}{\alpha}+c_{p-2}\right) r^{p+q-2}+\cdots \\
&+\left[\cdots\left(\left(\left(c_{p} \frac{p+q}{\alpha}+c_{p-1}\right) \frac{p+q-1}{\alpha}+c_{p-2}\right) \frac{p+q-2}{\alpha}+c_{p-3}\right) \cdots\right. \\
&\left.+c_{0}\right] \frac{I_{q}}{1}
\end{aligned}
$$

The calculations can be further simplified by defining the set of coefficients

$$
\begin{aligned}
D_{p+q} & =c_{p} \\
D_{p+q-1} & =c_{p} \frac{p+q}{\alpha}+c_{p-1}, \\
D_{p+q-2} & =\left(\left(c_{p} \frac{p+q}{\alpha}+c_{p-1}\right) \frac{p+q-1}{\alpha}+c_{p-2}\right), \\
D_{q} & =\left(\cdots\left(c_{p} \frac{p+q}{\alpha}+c_{p-1}\right) \frac{p+q-1}{\alpha}+\cdots+c_{0}\right) .
\end{aligned}
$$

With this and equation (7) on $I_{q}$ we obtain

$$
\begin{aligned}
I= & -\frac{1}{\alpha} e^{-\alpha r}\left\{D_{p+q} r^{p+q}+\cdots+D_{p+q-k} r^{p+q-k}+\cdots+D_{q} r^{q}\right\} \\
& -\frac{1}{\alpha} e^{-\alpha r} D_{q}\left\{\frac{q}{\alpha} r^{q-1}+\cdots+\frac{q(q-k)}{\alpha_{k}} r^{q-k}+\cdots+\frac{q !}{\alpha^{q}}\right\} \\
& +\frac{D_{q} q !}{\alpha^{q+1}} .
\end{aligned}
$$

Or, in a more compact notation

$$
I=\frac{D_{q} q !}{\alpha^{q+1}}-\frac{1}{\alpha} e^{-\alpha r}\left\{D_{q} q ! \sum_{j=1}^{q} \frac{r^{q-j}}{\alpha^{j}(q-j) !}+\sum_{j=q}^{q+p} D_{j} r^{j}\right\} .
$$

Similarly, for the second integral in equation (3), we have

$$
t=-\lambda+1+l+l^{\prime}
$$

and instead of equation (7) 
(9)

$$
J_{\mu}=\int_{r}^{\infty} e^{-\alpha x} x^{\mu} d x=\frac{1}{\alpha} e^{-\alpha r} r^{\mu}+\frac{\mu}{\alpha} J_{\mu-1}, \quad \mu \geqq 1,
$$

$$
J_{0}=\frac{1}{\alpha} e^{-\alpha t}
$$

The final expression is

$$
J=\frac{1}{\alpha} e^{-\alpha r}\left\{D_{t}^{\prime} t ! \sum_{j=1}^{t} \frac{r^{t-j}}{\alpha^{j}(t-j) !}+\sum_{j=t}^{t+p} D_{j}^{\prime} r^{j}\right\},
$$

where the $D_{i}^{\prime}$ is defined like the $D_{i}$ with $q$ replaced by $t$.

The final expression for $y_{\lambda}$ is readily obtained from

$$
y_{\lambda}=K_{v v^{\prime}}\left\{\frac{I}{r^{\lambda+1}}+r^{\lambda} J\right\}
$$

We note that, as required, the first term from equation (8) takes care of the first term on the right-hand side of equation (2) whenever $i=j$ and $\lambda=0$. Also note that for a many electron-atom the above procedure can be easily modified by replacing the functions $P_{n l}(x)$ with the one-electron orbitals usually given in the form

$$
R(r)=\sum_{i} c_{i} r^{n-1} e^{-\alpha_{i} r}
$$

(see $[6])$.

3. Numerical Results. A simple FORTRAN code has been written to evaluate equation (11). The only input parameters required are the principal and angular momentum quantum numbers $n$ and $l$, the values of $\lambda$, and the grid point. The code was tested extensively for various combinations of $n, l$ and $\lambda$ and the results compared with those of a similar code based on a quadrature rule. The analytic method here described has proved to be invariably superior, being accurate to seven significant figures, whereas the quadrature procedure was usually accurate to four significant figures and for some of the higher values of $n$, to two figures only. Some selected examples are given below:

\begin{tabular}{ccccc} 
Function & \multicolumn{4}{c}{ Formula } \\
$y_{0}\left(P_{10}, P_{10}\right)$ & $r$ & Quadrature & (Equation 11) & Exact \\
$y_{1}\left(P_{10}, P_{31}\right)$ & 1.0 & $7.300178(-1)$ & $7.293294(-1)$ & $7.293294(-1)$ \\
& 0.625 & $5.328259(-2)$ & $5.305263(-2)$ & $5.305262(-2)$ \\
& 2.5 & $5.962321(-2)$ & $5.957444(-2)$ & $5.957444(-2)$ \\
& 10.0 & $5.174112(-3)$ & $5.175395(-3)$ & $5.175395(-3)$ \\
$y_{1}\left(P_{30}, P_{41}\right)$ & 37.0 & $3.773509(-4)$ & $3.774209(-4)$ & $3.774209(-4)$ \\
& 37.5 & $3.860894(-3)$ & $3.884487(-3)$ & $3.884489(-3)$
\end{tabular}

Lockheed Research Laboratories Palo Alto, California 811.

1. D. R. Hartree, The Calculation of Atomic Structures, Wiley, New York, 1957. MR 19,

2. P. G. Burke \& H. M. Schey, Phys. Rev., v. 126, 1962, p. 147.

3. S. Ormonde \& K. Smith, Note on the Inelastic Scattering of Low Energy Electrons by Hydrogen Atoms, LMSC 6-90-63-25 (unpublished).

4. I. C. Percival \& M. J. Seaton, Proc. Cambridge Philos. Soc., v. 53, 1957, p. 654.

5. L. I. Schiff, Quantum Mechanics, 2nd ed., McGraw-Hill, New York, 1955.

6. C. C. G. RoothaAN \& P. S. Kelly, Phys. Rev., v. 131, 1963, p. 131. 